\title{
P-glycoprotein and its role in drug-drug interactions
}

\section{SUMMARY}

Efflux transporters such as P-glycoprotein play an important role in drug transport in many organs. In the gut, P-glycoprotein pumps drugs back into the lumen, decreasing their absorption.

Drugs which induce P-glycoprotein, such as rifampicin, can reduce the bioavailability of some other drugs. Inhibitors of P-glycoprotein, such as verapamil, increase the bioavailability of susceptible drugs.

Many, but not all, of the drugs which are transported by P-glycoprotein are also metabolised by cytochrome P450 3A4.

Important substrates of P-glycoprotein include calcium channel blockers, cyclosporin, dabigatran etexilate, digoxin, erythromycin, loperamide, protease inhibitors and tacrolimus. Predicting clinically important interactions is difficult because of interindividual differences in drug transport.

\section{Introduction}

P-glycoprotein is one of the drug transporters that determine the uptake and efflux of a range of drugs. This process affects their plasma and tissue concentrations and ultimately their final effects.

P-glycoprotein functions as a transmembrane efflux pump, pumping its substrates from inside to outside the cell. Drugs which induce or inhibit P-glycoprotein can interact with other drugs handled by the pump.

\section{Pharmacology}

P-glycoprotein was first described in tumour cells. These cells had over-expression of P-glycoprotein which reduced the access of cytotoxic drugs. As this made the tumours resistant to various anticancer drugs, P-glycoprotein was also known as multidrug resistance protein 1. P-glycoprotein is also expressed in a variety of normal, non-tumorous tissues with excretory functions (small intestine, liver and kidney) and at blood-tissue barriers (blood-brain barrier, blood-testis barrier and placenta). ${ }^{2}$

Along with the cytochrome P450 (CYP) family of enzymes, concomitant expression of P-glycoprotein is believed to be an important evolutionary adaptation against potentially toxic substances. As an efflux transporter it limits the bioavailability of orally administered drugs by pumping them back into the lumen. This promotes drug elimination into the bile and urine and protects a number of tissues such as the brain, testis, placenta and lymphocytes. The substrates for P-glycoprotein are a broad variety of structurally diverse compounds. ${ }^{2}$

\section{Drug absorption}

The epithelial cell lining of the small intestine is not just a site for drug absorption, but also an important barrier to the absorption of xenobiotics. P-glycoprotein is found in the apical (luminal) membrane of the entire intestine from duodenum to rectum, with a high expression in the enterocytes of the small intestine. It reduces the oral availability of drugs that are its substrates. ${ }^{3,4}$

Like the enzymes involved in drug metabolism, substrates of P-glycoprotein can potentially act as inhibitors or inducers of its function. Inhibition of $\mathrm{P}$-glycoprotein can result in increased bioavailability of the susceptible drug. Induction of P-glycoprotein reduces the bioavailability.

\section{Drug distribution}

Once a drug has reached the systemic circulation, P-glycoprotein further limits penetration into a number of sensitive tissues. P-glycoprotein is also important for the blood-brain barrier as a defence against the penetration of toxins and drugs into the central nervous system. ${ }^{3}$

\section{Drug elimination}

P-glycoprotein has a modest role in drug elimination. It is expressed in the luminal membrane of proximal tubule cells in the kidneys. P-glycoprotein pumps drugs into the urine.

\section{Drug interactions}

P-glycoprotein is an important mediator of drug-drug interactions. ${ }^{3}$ The pharmacokinetics of a drug may

\section{Andrew Finch \\ Advanced trainee \\ Peter Pillans \\ Director \\ Associate professor ${ }^{2}$ \\ ${ }^{1}$ Clinical Pharmacology \\ Princess Alexandra Hospital \\ ${ }^{2}$ Southside Clinical School \\ The University of \\ Queensland \\ Brisbane}

\section{Key words}

digoxin, pharmacokinetics

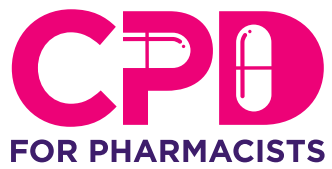

This article has a continuing professional development activity for pharmacists available at www.australianprescriber.com/ continuingprofessional development 
be altered when co-administered with compounds which inhibit or induce P-glycoprotein. ${ }^{3,5,6}$ Inhibitors include clarithromycin, erythromycin, ritonavir and verapamil. Inducers include rifampicin and St John's wort.

P-glycoprotein has a very wide substrate spectrum similar to CYP3A4. It is involved in the transport of drugs from different drug classes including:

- antineoplastic drugs e.g. docetaxel, etoposide, vincristine

- calcium channel blockers e.g. amlodipine

- calcineurin inhibitors e.g. cyclosporin, tacrolimus

- digoxin

- macrolide antibiotics e.g. clarithromycin

- protease inhibitors.

The substrates of P-glycoprotein can be further divided into drugs which are not metabolised in humans, such as digoxin, and those which are substrates of both P-glycoprotein and drugmetabolising enzymes, particularly CYP3A4. ${ }^{2,3}$ As many P-glycoprotein substrates are also substrates of CYP3A4 and because P-glycoprotein inhibitors are also inhibitors of CYP3A4, many drug-drug interactions are related to inhibition or induction of both P-glycoprotein and CYP3A4. Drugs which are 'objects' of such interactions include cyclosporin, tacrolimus and rivaroxaban. ${ }^{3}$

Enterocytes, like hepatocytes, simultaneously express the major drug-metabolising enzyme CYP3A4 and the efflux transporter P-glycoprotein. ${ }^{7}$ This creates a drug efflux-metabolism 'alliance', which increases the exposure of the drug to metabolism by CYP3A4 through repeated cycles of absorption and efflux. ${ }^{2}$ Modification of this active barrier function by concomitantly administered drugs contributes to altered absorption, increased interindividual differences in systemic drug concentrations and probably an increased risk of toxicity. ${ }^{4}$

Accurate prediction of potential drug-drug interactions through P-glycoprotein is complicated by pronounced interindividual differences in bioavailability. This also affects drugs that are not metabolised in humans (fexofenadine, digoxin). ${ }^{2,4} \mathrm{~A}$ better knowledge of the role of genetics in transporter expression and function will contribute to a better understanding of interindividual and interethnic differences in drug disposition and effects. ${ }^{2}$

\section{Loperamide}

P-glycoprotein is the most important drug transporter for reducing the entry of drugs into the central nervous system. The over-the-counter antidiarrhoeal medication loperamide is a potent opiate, but does not have opioid effects on the central nervous system at usual doses. This is because P-glycoprotein prevents transport across the blood-brain barrier.

Concomitant administration of loperamide and a potent P-glycoprotein inhibitor, such as verapamil, may be associated with respiratory depression. This potentially dangerous central nervous system effect from such a widely used and easily accessible drug is of great interest. It raises safety concerns but suggests that inhibiting P-glycoprotein could be a novel strategy to overcome the blood-brain barrier to increase drug delivery to the brain. ${ }^{8}$

\section{Digoxin}

Induction or inhibition of intestinal P-glycoprotein appears to be a major mechanism underlying drug interactions that lead to reduced or elevated digoxin concentrations. Rifampicin and St John's wort induce P-glycoprotein and thereby decrease concentrations of digoxin.

\section{HIV-1 protease inhibitors}

The effective treatment of HIV may be hindered by P-glycoprotein located in cell membranes. Potential mechanisms include the following:

- intestinal P-glycoprotein limits the absorption of HIV protease inhibitors

- HIV protease inhibitors are good P-glycoprotein substrates, ${ }^{9}$ so this limits their transfer across the blood-brain barrier, which can contribute to viral persistence and reduced effectiveness

- P-glycoprotein is also expressed in CD4 cells, the major target of anti-HIV drugs.

\section{Dabigatran}

As dabigatran etexilate is a substrate of P-glycoprotein, there is potential for drug interactions involving drugs acting on P-glycoprotein. Inhibitors of P-glycoprotein such as ketoconazole, amiodarone, verapamil, ticagrelor and clarithromycin may increase the peak plasma concentrations of dabigatran, and subsequently lead to a significantly increased risk of severe haemorrhage. ${ }^{10}$

\section{Conclusion}

P-glycoprotein is an efflux transporter pump present in many organs and plays an important role in drug transport. Expression of P-glycoprotein can have important effects on drug absorption, distribution and elimination. Although interactions with drug transporters can be clinically insignificant, an awareness of potential transporter-related drug-drug 
interactions is important. Central nervous system depression, undertreated HIV infection and transplant rejection are all possible outcomes if these interactions occur. Knowledge of these potential interactions in at-risk patient groups can help ensure the provision of safe and effective treatment. $\varangle$

\section{Conflict of interest: none declared}

\section{REFERENCES}

1. Thiebaut F, Tsuruo T, Hamada H, Gottesman MM, Pastan I, Willingham MC. Cellular localisation of the multidrug resistance gene product $\mathrm{P}$-glycoprotein in normal human tissues. Proc Natl Acad Sci USA 1987;84:7735-8.

2. Fromm MF. Importance of P-glycoprotein at blood-tissue barriers. Trends Pharmacol Sci 2004;25:424-9.

3. Konig J, Muller F, Fromm MF. Transporters and drug-drug interactions: Important determinants of drug disposition and effects. Pharmacol Rev 2013;65:944-66.

4. Igel S, Drescher S, Murdter T, Hofmann U, Heinkele G, Tegude $\mathrm{H}$, et al. Increased absorption of digoxin from the human jejunum due to inhibition of intestinal transportermediated efflux. Clin Pharmacokinet 2007:46:777-85.

5. Ho RH, Kim RB. Transporter and drug therapy: implications for drug disposition and disease. Clin Pharmacol Ther 2005;78:260-77.

6. International Transporter Consortium, Giacomini KM Huang SM, Tweedie DJ, Benet LZ, Brouwer KL, et al. Membrane transporters in drug development. Nat Rev Drug Discov 2010;9:215-36.
7. Canaparo R, Finnstrom N, Serpe L, Nordmark A, Muntoni E, Eandi $\mathrm{M}$, et al. Expression of CYP3A isoforms and P-glycoprotein in human stomach, jejunum and ileum. Clin Exp Pharmacol Physiol 2007;34:1138-44.

8. Sadeque AJM, Wandel C, He H, Shah S, Wood AJ. Increased drug delivery to the brain by P-glycoprotein inhibition. Clin Pharmacol Ther 2000;68:231-7.

9. Kim RB, Fromm MF, Wandel C, Leake B, Wood AJ, Roden DM, et al. The drug transporter P-glycoprotein limits oral absorption and brain entry of HIV-1 protease inhibitors. J Clin Invest 1998;101:289-94

10. Kawabata M, Yokoyama Y, Sasano T, Hachiya H, Tanaka Y, Yagishita A, et al. Bleeding events and activated partial thromboplastin time with dabigatran in clinical practice. J Cardiol 2013;62:121-6.

\section{FURTHER READING}

Pharmacology Weekly. Comprehensive drug reference table. San Antonio, TX: Pharmacology Weekly; 2012.

www.pharmacologyweekly.com/content/pages/drug-reference-

table-cyp-p450-ugt-enzymes-transporters-ab [cited 2014 Jul 11]

\section{New drugs}

\section{Afatinib}

Approved indication: non-small cell lung carcinoma

Giotrif (Boehringer Ingelheim)

$10 \mathrm{mg}, 30 \mathrm{mg}, 40 \mathrm{mg}$ and $50 \mathrm{mg}$ film-coated tablets Australian Medicines Handbook section 14.2.3

Like erlotinib (Aust Prescr 2006;29:53-5), gefitinib (Aust Prescr 2003;26:94-5) and crizotinib (Aust Prescr, published online 2014 Apr 16), afatinib is a tyrosine kinase inhibitor approved for advanced or metastatic non-small cell lung carcinoma. Afatinib irreversibly binds to the ErbB family of epidermal growth factor receptors - ErbB1 (epidermal growth factor receptor or EGFR), ErbB2 (human epidermal growth factor receptor 2 or HER2), ErbB3 and ErbB4. By blocking signalling from these molecules, afatinib slows down the growth and spread of tumour cells.

About $10 \%$ of Australian patients with non-small cell lung carcinoma have mutations in the EGFR gene. These are activating mutations which contribute to the malignant phenotype - the two most common are
Del 19 (deletion in exon 19) and L858R (point mutation in exon 21). Afatinib is only approved for patients who have tumours with these mutations.

An open-label phase III comparative trial assessed the efficacy of afatinib (40 mg once a day) as a first-line treatment in 345 patients with an activating mutation in their EGFR gene. A subgroup of 308 patients had the Del 19 or L858R mutation. After a median of 11 months, afatinib significantly prolonged median progression-free survival compared to chemotherapy (see Table). In the afatinib group, progression-free survival was 2.5 months longer in those with Del 19 or L858R mutations. Afatinib did not significantly prolong overall survival compared to chemotherapy. In a questionnaire about symptoms, the onset of cough $(p=0.007)$ and dyspnoea $(p=0.015)$ was significantly delayed with afatinib compared to chemotherapy. However, diarrhoea, dysphagia and sore mouth were reported to be worse. ${ }^{2}$

A phase II trial in lung adenocarcinoma found that median progression-free survival was slightly longer for patients who received afatinib first-line compared expressed in the following notes on newly approved products should be regarded as preliminary. At the time of publication, there may be limited published data and little experience in Australia of safety or efficacy. However, the Editorial Executive Committee believes that comments made in good faith at an early stage may still be of value. Before new drugs are prescribed, the Committee believes it is important that more detailed information is obtained from the manufacturer's approved product information, a drug information centre or some other appropriate source. 